\title{
HTML5-based tourism needs of users broadcast audio APP Design and Construction
}

\author{
Yujie Song ${ }^{1,}$, Sanxing $\mathrm{CaO}^{2, \mathrm{~b}}$ \\ ${ }^{1}$ School of Communication University of China, BeiJing 100042, China; \\ ${ }^{2}$ School of Communication University of China, BeiJing 100042, China;
}

Keywords: Audio, HTML5, custom spread, automatically match

\begin{abstract}
As in recent years, rapid development of mobile phone hardware and operating systems such as Android, ios continue to optimize the market potential of the mobile market is gradually excavated, which created hear Lang stretch of mobile terminal APP. How to promote APP and make targeted deployment according to customer needs in order to retain customers has become the focus, customized disseminate this paper based on HTML5 audio APP needs of different users.
\end{abstract}

\section{Introduction}

In recent years with the decline in the price of mobile hardware have more people have a good performance of mobile phones, which has sparked the development of the mobile terminal market, a wide range of APP after another dazzling difficult to choose. And in the context of media convergence of traditional broadcasting facing restructuring, its video and audio but also in mobile terminals such as mobile phones, seize share on pad. The reality is that video APP such as Youku, love Fantastic Art, and other long settled in the market, like in the big mouth sub-video company a share of really easy. But the audio APP market competition is not hot, the most popular audio APP is basically the Himalayas, its resource-rich loved by the users. Himalaya as an integrated audio APP advantage is comprehensive, but the disadvantage is integrated, that is not targeted content. Sometimes users need to find they want to know the audio is difficult, audio instead of text, can be retrieved in the search by keyword and presentation, audio only title keyword can not be retrieved based on its content.

\section{Demand survey}

According to market research, such as if a vast majority of audio APP devoted to young travelers willing to download a try. Perhaps people in tourism destination only a relatively shallow understanding, may not have the time, interest in the history and culture search on destination and other information, and others to the destination began to regret not gather relevant information on tourist destinations. But it can develop a specialized tourism-related APP do to meet this demand, when the user enters a destination based on the input matching APP related keywords return relevant content and also recommend relevant content and keywords such as accommodation, Snacks and historical and cultural characteristics. In this paper, based on the study of tourism HTML5 audio APP user needs and build design, produced by the interaction with the user HTML5 thus push different messages to different people selected according to.

\section{First, HTML}

HTML (Hypertext Markup Language) that is HTML is a page description language document, and HTML5 is the latest version of HTML. HTML has a more open, more secure, suitable for touch, energy and many other advantages, but also cross-system across devices, whether in Windows or MacOS can smooth run, which makes both computers or mobile phones can smooth access HTML5 website . Compared to traditional web development, based on HTML5 can easily construct similar to the traditional client software, web APP, desktop software easily into the Web area of expertise in the world, and used in this article the most important technology HTML5 CSS3 also by the 
technical level The innovation comes from. JavaScript is well known that the main role is to make the picture move, but based on JS animation in terms of both performance on the realization for developers is a huge challenge. CSS3 can easily achieve many effects, especially the key is that it will not affect the page semantics and performance. The main idea of this paper is based on the HTML5 do animation about a favorite tourist destination choice and preference of background selection based on user clicks from the database with the user point of interest audio and push.

\section{Second, the custom spread}

Customized communication, is based on feedback from the user's information, the specific information pushed to the user interface. To achieve this personal customization service is very necessary in the case of the user accurate user immediately push audio This APP will feel good in a very short period of time to seize the user. We should even told APP that generally rely on their own keywords to find the audio material so targeted is not strong, it is better for the user to go directly to the Himalayas that the comprehensive site search. Audio APP do tourism development of the most important is targeted to different people pushing different types of audio, allowing users to feel the needs of individuals can be solved APP, which is that it allows users to generate a sense of satisfaction.

Use HTML5 to make a movie, because there is a cross-platform HTML5 cross-system features, animations can be shared in microblogging, letter circle of friends, and of course the most important thing is to start the animation when the user first downloads the open APP. Animation can have on history, culture, unofficial relevant content for users to choose, in no particular destination user to select a particular item for audio when APP recommendations based on user feedback. When the user has the target directly in the search bar animation search for destinations, then APP do is give users recommend not only a description about the target, but also recommend related destinations such as food, customs and other audio information. For example, users search the Palace of audio, then APP will not only push information to the user about the Forbidden City Profile, humanities will push Beijing, the Forbidden City and the Emperor unofficial anecdotes and other information related to choice for users. At this time do a search for the audio data integrated push, which attract users is very large, because as a user using only very small amounts of the search to find a number of audio-related information and their favorite destination, which for the user of the APP Interested in are a substantial improvement.

\section{Third, the database automatically match}

The key to achieve custom spread APP is based on user feedback push information, and APP to do that is automatically matched against the database to the user based on the results push the audio match. In the database can give each audio few more fields, but different field represents the audio belong to different attributes. For example, you can probably give classified into four categories on the property, location, history and culture, attractions and cuisine. Or to the National Palace, for example, the Location field when the user enters the Forbidden City Palace Museum this data belongs to Beijing, the APP will match the same location of the audio data of Beijing to push, push and empathy will field the same history and culture of the audio data. After a judgment together with a selection decision statement, audio push under and object matching the best results, this approach in the general site search is very efficient. According to match the different fields of the final judgment of the output of the best results, this approach to the maximum extent possible and target the most relevant information matching push.

The key is to match the accuracy of the field is set, in the end the problem of how to set the fields in order to achieve the best match is to be considered. APP in the field of tourism set to fully consider the age of the person using the APP and its needs, according to the survey in front of the Forbidden City is willing to try most of the audio APP is young. In understanding the audience is young people after field selection focus is OK. More emphasis on young people when traveling where the fun, food and lodging, while older people may pay more attention to human history, in 
the case of relatively small proportion of older audiences accounted cultural history is not provided as a separate field. According to the preferences of young people attribute field divided into three categories, location, accommodation, food, location to determine where the audience recommended audio input information, and accommodation and food can push where accommodation and food according to the determined location. In the user's search is not the place but the food, when accommodation APP will automatically match the same reason and search for content related to the location and food, accommodation.

\section{Conclusions}

In this paper, through the input of information processing and automatic matched to the user associated audio recommended method that allows users to enter a destination in a one-time feedback on a variety of associated audio for users to choose, it is a good user a good impression for APP Users upgrading and promotion of friends. In H5 aspect allows users to select animation options, but also a database to match the user's selection. The core idea is to match the settings of APP through fields and push the audio, so that can improve the user experience to the maximum extent.

\section{References}

[1] Sai Zhan, Research on the identity of college students micro blog customized communication, G206,1009-5322 (2013) 05-0071-3

[2] Herbert A.Simon. The Quarterly Journal of Economics. 1955(1).Reference to a chapter in an edited book:

[3]Rheingold H. The Virtual Community: Homesteading on the Electronic

Frontier. Cambridge: MIT Press, 2000.

[4] PADUA Divid. Communication contention in APN list scheduling algorithm

[5] RHEINGOLD H, The virtual community-homesteading on the e-lectronic frontier (revised edition). . 20006, 